\title{
Stereotactic radiosurgery in the era of novel systemic therapy for lung cancer brain metastases
}

\author{
Sarah Mudra1', Shruti Bhandari², Prashant Tripathi ${ }^{3}$, Neal Dunlap ${ }^{4}$, Goetz Kloecker ${ }^{2}$ \\ 'University of Louisville School of Medicine, Louisville, KY 40202, USA. \\ ${ }^{2}$ Department of Internal Medicine, Division of Hematology and Medical Oncology, James Graham Brown Cancer Center, \\ University of Louisville School of Medicine, Louisville, KY 40202, USA. \\ ${ }^{3}$ Division of Infectious Disease, University of Louisville School of Medicine, Louisville, KY 40202, USA. \\ ${ }^{4}$ Department of Radiation Oncology, James Graham Brown Cancer Center, University of Louisville School of Medicine, Louisville, \\ KY 40202, USA.
}

Correspondence to: Dr. Goetz Kloecker, Department of Internal Medicine, Division of Hematology and Medical Oncology, James Graham Brown Cancer Center, University of Louisville School of Medicine, 529 South Jackson Street, Louisville, KY 40202, USA. E-mail: goetz.kloecker@louisville.edu

How to cite this article: Mudra S, Bhandari S, Tripathi P, Dunlap N, Kloecker G. Stereotactic radiosurgery in the era of novel systemic therapy for lung cancer brain metastases. J Cancer Metastasis Treat 2019;5:27.

http://dx.doi.org/10.20517/2394-4722.2019.09

Received: 15 Jan 2019 First Decision: 6 Feb 2019 Revised: 13 Feb 2019 Accepted: 20 Feb 2019 Published: 3 Apr 2019

Science Editor: Lucyna Kepka Copy Editor: Cai-Hong Wang Production Editor: Huan-Liang Wu

\begin{abstract}
The emergence of novel systemic therapies has spurred a dramatic paradigm shift in lung cancer treatment. Research has revealed greater intracranial efficacy in targeted agents and immune checkpoint inhibitors (ICI) compared to conventional chemotherapy. Concurrently, advances in stereotactic radiosurgery (SRS) have contributed to the increased use of this highly localized, minimally-invasive treatment modality for local tumor control. In this era of precision medicine, the combination of these novel agents and SRS demands further prospective exploration particularly as questions regarding their sequence of administration and the risk of neurotoxicity remain unanswered. Presently, although data are limited and largely retrospective, literature supports the concurrent administration of $\mathrm{ICl}$ and radiation, with no observed increases in immune-related adverse events or acute neurologic toxicities. In the case of patients with driver mutations, newer generations of tyrosine kinase inhibitors (TKI) display improved intracranial efficacy and are currently preferred alone upfront in patients with asymptomatic brain metastases (BM) due to lack of data. Evidence of combining TKI and SRS is limited with mixed results. In this review, we explore the evidence regarding the use of novel systemic agents and SRS for treatment of lung cancer BM. Clinical practice will continue to be refined as larger, prospective studies yield results.
\end{abstract}

Keywords: Lung cancer; stereotactic radiosurgery, brain metastasis, tyrosine kinase inhibitors, immunotherapy

(C) The Author(s) 2019. Open Access This article is licensed under a Creative Commons Attribution 4.0 International License (https://creativecommons.org/licenses/by/4.0/), which permits unrestricted use, sharing, adaptation, distribution and reproduction in any medium or format, for any purpose, even commercially, as long as you give appropriate credit to the original author(s) and the source, provide a link to the Creative Commons license, and indicate if changes were made. 


\section{INTRODUCTION}

Lung cancer remains the leading cause of cancer-related mortality ${ }^{[1]}$. Eighty-five percent of diagnoses represent non-small cell lung cancer (NSCLC ${ }^{[2]}$; the remaining $10 \%-14 \%$ are diagnoses of small cell lung cancer. Brain metastases (BM) are diagnosed in approximately $10 \%$ of lung cancers at time of diagnosis ${ }^{[3]}$ and approximately $40 \%-50 \%$ will be diagnosed with $\mathrm{BM}$ during the course of their disease $\mathrm{e}^{[3,4]}$. BM in lung cancer are known to be associated with poor prognosis. Historically, standard treatment for BM from lung cancer involves neurosurgical resection ${ }^{[5]}$, radiotherapy and/or chemotherapy $\mathrm{y}^{[5,6]}$.

Recent advances in NSCLC, including molecular analysis, matched targeted therapies and immunotherapy, has altered the standard of care. These novel approaches have shifted the paradigm in lung cancer ${ }^{[7]}$ and improved median overall survival in lung cancer patients with $\mathrm{BM}^{[7,8]}$. Survival now ranges between 9-15 months and can be as high as 46 months in patients with favorable prognostic factors such as good performance status and epidermal growth factor receptor (EGFR) and/or anaplastic lymphoma kinase (ALK) positivity $^{[9]}$. In addition, over the past decade, standard of care in radiotherapy for BM has increasingly favored SRS ${ }^{[10,11]}$. Precise delivery of high-dose radiation localized to the tumor results in higher local tumor control $^{[10]}$ and fewer side effects such as neurocognitive damage ${ }^{[11]}$ compared to whole brain radiotherapy (WBRT).

The trifold advancement in targeted therapies, immunotherapy and SRS has revolutionized the treatment of BM in lung cancer. Employing these novel therapies, lung cancer patients are living longer, becoming more likely to develop brain metastases. Certainly, the use of these therapies - either individually or in combination - is anticipated for the treatment of lung cancer BM.

In this review, we discuss the current evidence regarding the use of SRS employed alone and in combination with novel therapies for treatment of lung cancer BM.

\section{BRAIN METASTASIS IN LUNG CANCER LACKING A DRIVER MUTATION}

\section{SRS}

SRS, typically delivered in a single fraction, serves as a key modality for delivering high-dose radiation to smaller target sites (usually $<3 \mathrm{~cm}$ ) ${ }^{[12]}$, sparing adjacent structures from exposure and mitigating the harmful effects of radiation ${ }^{[11]}$.

For single BM, SRS has demonstrated efficacy and safety. Therefore, both surgical resection plus postoperative radiation and SRS alone are reasonable options and treatment should be individualized as comparative data is lacking ${ }^{[13]}$. Surgery plus postoperative radiation is preferred for a single, large and symptomatic BM to allow for decompression, lower morbidity and higher local control ${ }^{[13]}$. One small retrospective study found similar survival when comparing surgical resection $v$ s. SRS for solitary $\mathrm{BM}^{[14]}$. However, more local recurrence was demonstrated in the surgery group ${ }^{[14]}$.

For surgically-resected brain metastases, post-operative SRS (post-SRS) is the current standard of care. Some concerns with post-SRS include radiation necrosis and leptomeningeal disease (LMD) recurrence. The hypothesis behind LMD recurrence is the intra-operative seeding of viable tumor cells, which is supported by a study where post-SRS demonstrated higher rates of LMD compared to adjuvant WBRT ${ }^{[15]}$. A new approach, pre-operative SRS (pre-SRS), is being evaluated as a potential method to decrease radiation necrosis and LMD. Potential benefits of pre-SRS include: (1) better local tumor control through improved delineation when contouring an intact metastasis compared to an irregularly-shaped surgical cavity; (2) reduced risk of radiation necrosis, as there is no need to treat surrounding brain tissue and the majority of the treated BM will be resected; (3) reduced risk of LMD as a result of a potential sterilizing effect via the intraoperative seeding of treated tumor cells; and (4) the potential to treat more patients, as with post- 
SRS some patients are lost to follow-up. A potential disadvantage includes reduced wound healing ${ }^{[15]}$. Asher et al. ${ }^{[16]}$ evaluated pre-SRS in 47 patients, demonstrating its safety and efficacy with local control rates of $85.6 \%$ at 12 months. One retrospective study evaluating pre- and post-SRS cohorts of $180 \mathrm{BM}$ patients (including approximately $40 \%$ with NSCLC) showed similar rates of local recurrence and overall survival. However, pre-SRS was associated with significantly reduced rates of radiation necrosis and $\mathrm{LMD}^{[17]}$. Clinical trials currently comparing pre-SRS and post-SRS in BM include NCT03741673 and NCT03750227.

SRS plays a major role in patients with multiple small $\mathrm{BM}(<3 \mathrm{~cm})$ or surgically inaccessible BM. Younger lung cancer patients of high Karnofsky performance status with limited BM and a low burden of extracranial disease may derive the most benefit from SRS ${ }^{[10,18]}$. Generally, SRS use has been limited to patients with few, small, easily radio-accessible $\mathrm{BM}^{[2]}$. Although a multi-center retrospective analysis demonstrated a survival advantage for patients treated with SRS possessing fewer than four BM ( $n=189$ for NSCLC) compared to WBRT (adjusted HR for NSCLC, 0.58; 95\%CI: 0.38-0.87; $P=0.01)^{[19]}$, Yamamoto and colleagues recently evaluated SRS alone in 1,194 patients with up to ten lesions (largest tumor $<10 \mathrm{~mL}$ in volume and $<3 \mathrm{~cm}$ in longest diameter; total cumulative volume $\leq 15 \mathrm{~mL})^{[20]}$. Their group found no difference in overall survival (10.8 months) or treatment-related adverse events (9\%) between patients with two to four tumors and patients with five to ten tumors ${ }^{[20]}$. These results suggest expanding SRS for the treatment of patients with up to ten $\mathrm{BM}^{[2,5]}$. Further data propose total $\mathrm{BM}$ volume as potentially more significant than the total number of $\mathrm{BM}^{[10]}$.

\section{Adverse effects of SRS}

Overall, the adverse effects of SRS are consistent with known toxicities of intracranial irradiation. Both acute (developing over weeks to months) and late-onset (developing over months to years) toxicities may result. Certainly, the risk, severity and incidence of radiation-induced toxicities is highly dependent on the site, dose, fractionation and volume of tissue irradiated along with the patient's comorbidities ${ }^{[21]}$. Acute toxicities are uncommon and include nausea, headache, dizziness, seizure or new transient focal deficits ${ }^{[22]}$. Patients are usually treated with a short course of glucocorticoids.

The most common delayed adverse effect of SRS treatment to BM is radiation necrosis, occurring in approximately $5 \%-10 \%$ of patients ${ }^{[23]}$. However, this risk rapidly increases with increasing BM size and/ or volume as well as with a history of radiation to same lesion ${ }^{[21,23-25]}$. A study suggests employing multifractionated SRS in three to five fractions rather than single fraction SRS as a means of decreasing the risk of radiation necrosis and improving local control ${ }^{[26]}$. The use of fractionated SRS also allows for the safe treatment of larger BM $(>3 \mathrm{~cm})^{[2,2,2]}$. Data on long-term effects of SRS on neurocognition is limited but reassuring ${ }^{[25]}$.

\section{SRS in combination with immunotherapy}

Immune checkpoint inhibitors (ICIs) have become a routine part of the treatment of advanced NSCLC lacking a driver mutation, administered with chemotherapy doublet or alone in patients with $\geq 50 \%$ expression of programmed cell death-ligand $1(\mathrm{PD}-\mathrm{L} 1)^{[8]}$. Most patients with advanced NSCLC and BM are eligible to receive ICIs - either alone or with chemotherapy.

Concerns to using ICIs to treat BM include: (1) pseudo-progression with the potential of symptom aggravation; and (2) steroid use for symptomatic BM which may reduce ICI activity, as demonstrated by a decreased objective brain response with the addition of steroids during ICI treatment in melanoma ${ }^{[28]}$. Present data on ICIs for BM from advanced NSCLC is limited. Most ICI clinical trials in NSCLC excluded patients with untreated or unstable BM, yet included stable and treated BM, comprising 6\%-17\% of included patients $^{[29]}$. Overall, in the small subgroups of BM patients included in these trials, ICI appears safe ${ }^{[29]}$. However, outcomes were mixed as some trials demonstrated benefit over chemotherapy and others did not $^{[29]}$. A rationale for positive response to ICIs includes the inflammatory microenvironment of BM, with 
the presence of significant tumor-infiltrating lymphocytes (TILs). In a series of $116 \mathrm{BM}$ specimens (including 61 from NSCLC BM), more than 50\% of all specimens had dense TIL infiltration - also associated with improved survival ${ }^{[30]}$.

Radiation therapy (RT) also induces an antitumor immune response by upregulating PD-L1 and inflammatory cytokines as well as facilitating T-cell infiltration ${ }^{[31]}$. Localized RT may induce an abscopal effect, which reflects the regression of non-irradiated metastatic lesions due to systemic anti-tumor response. The dose and fractionation of RT plays a role in its effects on the immune system. Schaue et al ${ }^{[32]}$ found that fractionated treatment with medium-sized radiation doses of 7.5 Gy per fraction yielded the best tumor control and antitumor immune responses. Dewan et al.$^{[33]}$ showed that $5 \times 6$ Gy and $3 \times 8$ Gy protocols of RT were more effective in inducing immune-mediated abscopal effects than a single ablative dose of 20 Gy when combined with an anti-CTLA-4 antibody. These preclinical data suggest a better systemic anti-tumor effect with hypofractionated (e.g., fractionated SRS) than conventional RT. Most data regarding the abscopal effect are from treating systemic disease; if the same impact occurs in the treatment of BM is uncertain. Although based on small studies, there is growing evidence in favor of an abscopal effect when treating $\mathrm{BM}^{[34]}$.

RT alone is a poor inducer of immune-mediated local and abscopal responses; but, evidence suggests these responses are enhanced by combining radiation with $\mathrm{ICI}^{[31,35,36]}$. Although data regarding the combination of ICI and radiotherapy in BM due to NSCLC are limited and mostly retrospective, data indicate that combining ICI and radiation in BM is safe with similar adverse events ${ }^{[37,38]}$ and support a concurrent administration of ICI with radiation over a sequential administration ${ }^{[38]}$. While most studies demonstrated similar adverse events with combination ICI-radiotherapy, the role of ICI in radiation necrosis remains controversial. One retrospective study with 61\% NSCLC patients showed that the incidence of symptomatic radiation necrosis after stereotactic radiation was higher in patients who received ICI - especially those with melanoma ${ }^{[39]}$. Other retrospective studies including patients with NSCLC and BM did not report a higher indicence of radiation necrosis with combination ICI-radiotherapy ${ }^{[37,38,40]}$.

Many questions regarding the combination of ICI and RT remain unanswered including the optimal timing, the impact of steroids and neurotoxicity. Questions such as these should be investigated through prospective trials. Current clinical trials evaluating the combination of ICI and radiation therapy in NSCLC BM include NCT02978404 (Nivolumab + SRS), NCT02858869 (Pembrolizumab + SRS) and NCT02696993 (Nivolumab + SRS/WBRT and Nivolumab + Ipilimumab + SRS/WBRT). These phase I and phase II trials include other malignancies and are expected to finish by 2020 .

\section{BRAIN METASTASIS IN LUNG CANCER WITH DRIVER MUTATIONS}

In NSCLC, sensitizing EGFR mutations are found in $10 \%$ of Caucasians as well as up to $50 \%$ of Asians ${ }^{[41]}$. ALK-rearrangement is found in $2 \%-7 \%{ }^{[42]}$ and ROS proto-oncogene 1 (ROS1) occurs in $1 \%-2 \%$ of patients ${ }^{[43]}$. The incidence of BM is higher in patients with driver mutations. The rates of BM present at diagnosis is $24.2 \%$ and $23.8 \%$ in EGFR-mutated and ALK-rearranged lung cancers, respectively, and increasing to $>45 \%$ of patients at three years post-diagnosis ${ }^{[44]}$. ROS1-rearranged NSCLC also has a high incidence of BM (36\%) and is the common first site of progression ${ }^{[45]}$.

First- (erlotinib, gefinitib) and second-generation (afatinib) anti-EGFR TKI have demonstrated improved survival and brain response rates of over $50 \%$ in EGFR-mutated patients compared to EGFR wildtype patients ${ }^{[5]}$. The third-generation anti-EGFR TKI, osimertinib, demonstrated an even higher rate of intracranial response ( $91 \%$ vs. $68 \%$ in patients with measurable BM lesions), a lower rate of central nervous system (CNS) progression and longer progression-free survival when compared to first generation anti-EGFR TKI in the FLAURA trial ${ }^{[46,47]}$. Though first- and second-generation anti-EGFR TKI have activity in patients with $\mathrm{BM}$, these agents have a much lower intracranial concentration as compared with osimertinib ${ }^{[5]}$. Given 
the high intracranial activity of osimertinib, it is preferred as the initial therapy for patients presenting with asymptomatic BM in EGFR-mutated NSCLC. Osimertinib has not yet been evaluated with SRS, but it is reasonable to use SRS in the case of isolated intracranial progression and continue to use osimertinib. A clinical trial is open to evaluate osimertinib with or without SRS for EGFR-mutated NSCLC with BM (NCT03497767). The trial is expected to be completed in 2022.

If osimertinib is unavailable, first- or second-generation anti-EGFR TKI can be used in patients with asymptomatic BM, but not alone. Preclinical studies have shown a sensitizing effect of radiotherapy on EGFR expression and an enhanced radiation response through the inhibition of $\operatorname{EGFR}^{[48,49]}$. Although limited, clinical data is not yet reflective of preclinical data, but shows promise for future trials. A meta-analysis including 363 patients and another retrospective study of 351 patients with EGFR-mutated NSCLC (treated with first- and second-generation anti-EGFR TKI) suggested upfront intracranial radiation demonstrates better overall survival ${ }^{[50,51]}$. SRS followed by anti-EGFR TKI resulted in the longest overall survival, with WBRT followed by anti-EGFR TKI demonstrating intermediate overall survival and anti-EGFR TKI followed by SRS or WBRT at intracranial progression resulting in the shortest median overall survival ${ }^{[50,51]}$. Based on these data, for patients with asymptomatic BM started on a first- or second-generation anti-EGFR TKI, upfront SRS is appropriate - particularly given its better outcomes compared to delaying radiation. The concurrent use of anti-EGFR TKI with SRS or WBRT is more controversial, demonstrating mixed results. A phase III study found decreased overall survival with WBRT + SRS + erlobinib compared to WBRT + SRS ${ }^{[52]}$. Another retrospective study found similar survival in patients receiving a concurrent administration of radiation + anti-EGFR TKI $v$ s. patients receiving radiation followed by anti-EGFR $\mathrm{TKI}^{[53]}$. At this point, due to the absence of data, we recommend stopping TKI administration during SRS treatment and resuming after completion.

ALK-directed TKIs have also demonstrated intracranial activity. In a retrospective analysis, the firstgeneration ALK inhibitor, crizotinib, showed an intracranial disease control rate of $56 \%$ in untreated BM and $62 \%$ in previously treated $\mathrm{BM}^{[54]}$. Despite this intracranial efficacy, approximately $20 \%$ of patients progressing on crizotinib developed $\mathrm{BM}^{[54]}$. Second-generation ALK-directed TKIs (ceritinib, alectinib, brigatinib) have better intracranial efficacy. Alectinib was compared to crizotinib in two phase III trials (J-ALEX and ALEX) and showed improved survival and superior CNS activity with an incidence rate of CNS progression at 12 months of $4.6 \%$ with alectinib compared to $32 \%$ with crizotinib in patients without BM at baseline ${ }^{[55-57]}$. Brigatinib is also superior to crizotinib in the frontline setting, as seen in the ALTA trial, with an intracranial response rate of $78 \%$ with brigatinib vs. $28 \%$ with crizotinib ${ }^{[58]}$. Crizotinib, ceritinib, alectinib and brigatinib are all approved as first-line therapies for ALK-positive advanced NSCLC. However, alectinib is preferred per National Comprehensive Cancer Network guidelines ${ }^{[8]}$. Alectinib alone is appropriate in asymptomatic BM.

In patients who progress on crizotinib, ceritinib, alectinib or brigatinib are all appropriate as they have not been directly compared in terms of BM efficacy. In a small case series, alectinib also showed intracranial response in ceritinib-resistant patients ${ }^{[59]}$. In the setting of alectinib-resistant disease, options include switching to lorlatinib, which has documented CNS activity in patients who have failed second-generation inhibitors ${ }^{[60]}$ or local therapy (such as SRS) if only oligometastatic disease is present ${ }^{[61]}$.

Crizotinib is the standard first-line treatment for patients with ROS1-rearranged NSCLC ${ }^{[8]}$, but as previously discussed, it has poor intracranial activity. Other approved TKIs include ceritinib and lorlatinib that demonstrate better intracranial activity ${ }^{[62,63]}$. There are no data available on the combination of radiation therapy and TKI in ROS1-rearranged NSCLC.

In one study, patients with EGFR-mutated or ALK-rearranged NSCLC who had oligo-progression on erlotinib or crizotinib were considered for local ablative therapy to the sites of progression and continuation 
of the TKI ${ }^{[64]}$. The TKI was stopped on the days of radiation and restarted with the same dose ${ }^{[64]}$. Twentyfive of 51 patients who progressed were deemed suitable for local therapy and most received stereotactic radiation ${ }^{[64]}$. Ten of the 25 patients had intracranial progression ${ }^{[64]}$. Post local ablative therapy, the median progression-free survival was six months ${ }^{[64]}$. Minimal grade three and four adverse events were seen ${ }^{[64]}$. Another study of ALK-rearranged NSCLC showed that combining stereotactic radiation with crizotinib is safe and can achieve durable control, although the study only included extracranial sites of progression ${ }^{[65]}$. A retrospective study showed prolonged overall survival (49.5 months) of NSCLC patients with BM when treated with ALK-directed TKI therapy and brain radiation ${ }^{[66]}$. In the ALEX trial, patients with previous radiation to $\mathrm{BM}$ had higher intracranial response rates ( $86 \%$ vs. $79 \%$ ) compared with patients without prior radiotherapy ${ }^{[57]}$. In summary, data on the safety and outcome of combining radiation with ALK-directed TKIs is limited and favors SRS over WBRT.

An ongoing clinical trial is evaluating ALK inhibitors and other targeted therapies in combination with stereotactic brain treatment in patients with stage IV oncogene-driven (EGFR, ALK, or ROS1) NSCLC (NCT02314364).

\section{CONCLUSION}

The development of novel, targeted agents and immunotherapy has advanced the systemic treatment of lung cancer. These therapeutics demonstrate far greater intracranial efficacy than conventional chemotherapy - transformative for BM treatment. However, this paradigm shift in treatment warrants the careful consideration of systemic therapy as a frontline approach. While SRS remains an important aspect of the management of $\mathrm{BM}$, its role combined with novel systemic therapies is largely unclear. Limited available evidence suggests combination is safe with favorable outcomes, but the sequence of administration remains uncertain. Many clinical trials are underway that aim to further address these questions. As the results of these studies emerge, clinicians will gain further evidence-based insight into the clinical management of patients with lung cancer BM.

\section{DECLARATIONS}

\section{Authors' contributions}

Made substantial contributions to the research, writing and editing of the manuscript: All authors

Availability of data and materials

Not applicable.

\section{Financial support and sponsorship}

None.

\section{Conflicts of interest}

All authors declared that there are no conflicts of interest.

\section{Ethical approval and consent to participate}

Not applicable.

\section{Consent for publication}

Not applicable.

\section{Copyright}

(c) The Author(s) 2019. 


\section{REFERENCES}

1. Siegel RL, Miller KD, Jemal A. Cancer statistics, 2019. CA Cancer J Clin 2019;69:7-34.

2. McGranahan T, Nagpal S. A neuro-oncologist's perspective on management of brain metastases in patients with EGFR mutant non-small cell lung cancer. Curr Treat Options Oncol 2017;18:22.

3. Waqar SN, Samson PP, Robinson CG, Bradley J, Devarakonda S, et al. Non-small-cell lung cancer with brain metastasis at presentation. Clin Lung Cancer 2018;19:e373-e9.

4. Quan AL, Videtic GM, Suh JH. Brain metastases in small cell lung cancer. Oncology (Williston Park) 2004;18:961-72; discussion 74, 7980,87 .

5. Bui N, Woodward B, Johnson A, Husain H. Novel treatment strategies for brain metastases in non-small-cell lung cancer. Curr Treat Options Oncol 2016;17:25.

6. Wang YY, Wang YS, Liu T, Yang K, Yang GQ, et al. Efficacy study of CyberKnife stereotactic radiosurgery combined with CIK cell immunotherapy for advanced refractory lung cancer. Exp Ther Med 2013;5:453-6.

7. Thapa B, Lauko A, Desai K, Venur VA, Ahluwalia MS. Novel systemic treatments for brain metastases from lung cancer. Curr Treat Options Neurol 2018;20:48.

8. NCCN Clinical Practice Guidelines in Oncology. Non-Small Cell Lung Cancer, Version 2.2019. NCCN Clinical Practice Guidelines in Oncology (NCCN Guidelines); 2018.

9. Sperduto PW, Yang TJ, Beal K, Pan H, Brown PD, et al. Estimating survival in patients with lung cancer and brain metastases: an update of the graded prognostic assessment for lung cancer using molecular markers (Lung-molGPA). JAMA Oncol 2017;3:827-31.

10. Bergsma DP, Salama JK, Singh DP, Chmura SJ, Milano MT. Radiotherapy for oligometastatic lung cancer. Front Oncol 2017;7:210.

11. Aly Z, Peereboom DM. Combination of radiotherapy and targeted agents in brain metastasis: an update. Curr Treat Options Neurol 2016;18:32.

12. Linskey ME, Andrews DW, Asher AL, Burri SH, Kondziolka D, et al. The role of stereotactic radiosurgery in the management of patients with newly diagnosed brain metastases: a systematic review and evidence-based clinical practice guideline. J Neurooncol 2010;96:45-68.

13. Fuentes R, Osorio D, Exposito Hernandez J, Simancas-Racines D, Martinez-Zapata MJ, et al. Surgery versus stereotactic radiotherapy for people with single or solitary brain metastasis. Cochrane Database Syst Rev 2018;8:Cd012086.

14. O’Neill BP, Iturria NJ, Link MJ, Pollock BE, Ballman KV, et al. A comparison of surgical resection and stereotactic radiosurgery in the treatment of solitary brain metastases. Int J Radiat Oncol Biol Phys 2003;55:1169-76.

15. Routman DM, Yan E, Vora S, Peterson J, Mahajan A, et al. Preoperative stereotactic radiosurgery for brain metastases. Front Neurol 2018;9:959

16. Asher AL, Burri SH, Wiggins WF, Kelly RP, Boltes MO, et al. A new treatment paradigm: neoadjuvant radiosurgery before surgical resection of brain metastases with analysis of local tumor recurrence. Int J Radiat Oncol Biol Phys 2014;88:899-906.

17. Patel KR, Burri SH, Asher AL, Crocker IR, Fraser RW, et al. Comparing preoperative with postoperative stereotactic radiosurgery for resectable brain metastases: A multi-institutional analysis. J Neurooncol 2017;131:611-8.

18. Sperduto PW, Kased N, Roberge D, Xu Z, Shanley R, et al. Summary report on the graded prognostic assessment: an accurate and facile diagnosis-specific tool to estimate survival for patients with brain metastases. J Clin Oncol 2012;30:419-25.

19. Halasz LM, Uno H, Hughes M, D'Amico T, Dexter EU, et al. Comparative effectiveness of stereotactic radiosurgery versus whole-brain radiation therapy for patients with brain metastases from breast or non-small cell lung cancer. Cancer 2016;122:2091-100.

20. Yamamoto M, Serizawa T, Shuto T, Akabane A, Higuchi Y, et al. Stereotactic radiosurgery for patients with multiple brain metastases (JLGK0901): a multi-institutional prospective observational study. Lancet Oncol 2014;15:387-95.

21. Sneed PK, Mendez J, Vemer-van den Hoek JG, Seymour ZA, Ma L, et al. Adverse radiation effect after stereotactic radiosurgery for brain metastases: incidence, time course, and risk factors. J Neurosurg 2015;123:373-86.

22. Chin LS, Lazio BE, Biggins T, Amin P. Acute complications following Gamma Knife radiosurgery are rare. Surg Neurol 2000;53:498502; discussion 502 .

23. Miller JA, Bennett EE, Xiao R, Kotecha R, Chao ST, et al. Association between radiation necrosis and tumor biology after stereotactic radiosurgery for brain metastasis. Int J Radiat Oncol Biol Phys 2016;96:1060-9.

24. Shaw E, Scott C, Souhami L, Dinapoli R, Kline R, et al. Single dose radiosurgical treatment of recurrent previously irradiated primary brain tumors and brain metastases: final report of RTOG protocol 90-05. Int J Radiat Oncol Biol Phys 2000;47:291-8.

25. Yamamoto M, Serizawa T, Higuchi Y, Sato Y, Kawagishi J, et al. A multi-institutional prospective observational study of stereotactic radiosurgery for patients with multiple brain metastases (JLGK0901 Study Update): Irradiation-related complications and long-term maintenance of mini-mental state examination scores. Int J Radiat Oncol Biol Phys 2017;99:31-40.

26. Minniti G, Scaringi C, Paolini S, Lanzetta G, Romano A, et al. Single-fraction versus multifraction (3 x 9 Gy) stereotactic radiosurgery for large $(>2 \mathrm{~cm}$ ) brain metastases: a comparative analysis of local control and risk of radiation-induced brain necrosis. Int J Radiat Oncol Biol Phys 2016;95:1142-8.

27. Masucci GL. Hypofractionated radiation therapy for large brain metastases. Front Oncol 2018;8:379.

28. Margolin K, Ernstoff MS, Hamid O, Lawrence D, McDermott D, et al. Ipilimumab in patients with melanoma and brain metastases: an open-label, phase 2 trial. Lancet Oncol 2012;13:459-65.

29. El Rassy E, Botticella A, Kattan J, Le Pechoux C, Besse B, et al. Non-small cell lung cancer brain metastases and the immune system: from brain metastases development to treatment. Cancer Treat Rev 2018;68:69-79.

30. Berghoff AS, Fuchs E, Ricken G, Mlecnik B, Bindea G, et al. Density of tumor-infiltrating lymphocytes correlates with extent of brain edema and overall survival time in patients with brain metastases. Oncoimmunology 2015;5:e1057388.

31. Frey B, Rubner Y, Kulzer L, Werthmoller N, Weiss EM, et al. Antitumor immune responses induced by ionizing irradiation and further immune stimulation. Cancer Immunol Immunother 2014;63:29-36. 
32. Schaue D, Ratikan JA, Iwamoto KS, McBride WH. Maximizing tumor immunity with fractionated radiation. Int J Radiat Oncol Biol Phys 2012;83:1306-10.

33. Dewan MZ, Galloway AE, Kawashima N, Dewyngaert JK, Babb JS, et al. Fractionated but not single-dose radiotherapy induces an immune-mediated abscopal effect when combined with anti-CTLA-4 antibody. Clin Cancer Res 2009;15:5379-88.

34. Chicas-Sett R, Morales-Orue I, Rodriguez-Abreu D, Lara-Jimenez P. Combining radiotherapy and ipilimumab induces clinically relevant radiation-induced abscopal effects in metastatic melanoma patients: a systematic review. Clin Transl Radiat Oncol 2017;9:5-11.

35. Knisely JP, Yu JB, Flanigan J, Sznol M, Kluger HM, et al. Radiosurgery for melanoma brain metastases in the ipilimumab era and the possibility of longer survival. J Neurosurg 2012;117:227-33.

36. Ngwa W, Irabor OC, Schoenfeld JD, Hesser J, Demaria S, et al. Using immunotherapy to boost the abscopal effect. Nat Rev Cancer 2018;18:313-22.

37. Hubbeling HG, Schapira EF, Horick NK, Goodwin KEH, Lin JJ, et al. Safety of combined pd-1 pathway inhibition and intracranial radiation therapy in non-small cell lung cancer. J Thorac Oncol 2018;13:550-8.

38. Chen L, Douglass J, Kleinberg L, Ye X, Marciscano AE, et al. Concurrent immune checkpoint inhibitors and stereotactic radiosurgery for brain metastases in non-small cell lung cancer, melanoma, and renal cell carcinoma. Int J Radiat Oncol Biol Phys 2018;100:916-25.

39. Martin AM, Cagney DN, Catalano PJ, Alexander BM, Redig AJ, et al. Immunotherapy and symptomatic radiation necrosis in patients with brain metastases treated with stereotactic radiation. JAMA Oncol 2018;4:1123-4.

40. Ahmed Kamran AK, Kim S, Arrington J, Naghavi AO, Dilling TJ. Outcomes targeting the PD-1/PD-L1 axis in conjunction with stereotactic radiation for patients with non-small cell lung cancer brain metastases. J Neurooncol 2017;133:331-8.

41. Hirsch FR, Bunn PA Jr. EGFR testing in lung cancer is ready for prime time. Lancet Oncol 2009;10:432-3.

42. Kwak EL, Bang YJ, Camidge DR, Shaw AT, Solomon B, et al. Anaplastic lymphoma kinase inhibition in non-small-cell lung cancer. N Engl J Med 2010;363:1693-703.

43. Dugay F, Llamas-Gutierrez F, Gournay M, Medane S, Mazet F, et al. Clinicopathological characteristics of ROS1- and RET-rearranged NSCLC in caucasian patients: data from a cohort of 713 non-squamous NSCLC lacking KRAS/EGFR/HER2/BRAF/PIK3CA/ALK alterations. Oncotarget 2017;8:53336-51.

44. Rangachari D, Yamaguchi N, VanderLaan PA, Folch E, Mahadevan A, et al. Brain metastases in patients with EGFR-mutated or ALKrearranged non-small-cell lung cancers. Lung Cancer 2015;88:108-11.

45. Patil T, Smith DE, Bunn PA, Aisner DL, Le AT, et al. The incidence of brain metastases in stage IV ROS1-rearranged non-small cell lung cancer and rate of central nervous system progression on crizotinib. J Thorac Oncol 2018;13:1717-26.

46. Soria JC, Ohe Y, Vansteenkiste J, Reungwetwattana T, Chewaskulyong B, et al. Osimertinib in untreated EGFR-Mutated advanced nonsmall-cell lung cancer. N Engl J Med 2018;378:113-25.

47. Reungwetwattana T, Nakagawa K, Cho BC, Cobo M, Cho EK, et al. CNS response to osimertinib versus standard epidermal growth factor receptor tyrosine kinase inhibitors in patients with untreated EGFR-Mutated advanced non-small-cell lung cancer. J Clin Oncol 2018; doi: 10.1200/JCO.2018.78.3118.

48. Akimoto T, Hunter NR, Buchmiller L, Mason K, Ang KK, et al. Inverse relationship between epidermal growth factor receptor expression and radiocurability of murine carcinomas. Clin Cancer Res 1999;5:2884-90.

49. Chinnaiyan P, Huang S, Vallabhaneni G, Armstrong E, Varambally S, et al. Mechanisms of enhanced radiation response following epidermal growth factor receptor signaling inhibition by erlotinib (Tarceva). Cancer Res 2005;65:3328-35.

50. Soon YY, Leong CN, Koh WY, Tham IW. EGFR tyrosine kinase inhibitors versus cranial radiation therapy for EGFR mutant non-small cell lung cancer with brain metastases: a systematic review and meta-analysis. Radiother Oncol 2015;114:167-72.

51. Magnuson WJ, Lester-Coll NH, Wu AJ, Yang TJ, Lockney NA, et al. Management of brain metastases in tyrosine kinase inhibitornaive epidermal growth factor receptor-mutant non-small-cell lung cancer: A retrospective multi-institutional analysis. J Clin Oncol 2017;35:1070-7.

52. Sperduto PW, Wang M, Robins HI, Schell MC, Werner-Wasik M, et al. A phase 3 trial of whole brain radiation therapy and stereotactic radiosurgery alone versus WBRT and SRS with temozolomide or erlotinib for non-small cell lung cancer and 1 to 3 brain metastases: Radiation Therapy Oncology Group 0320. Int J Radiat Oncol Biol Phys 2013;85:1312-8.

53. Wang W, Song Z, Zhang Y. Efficacy of brain radiotherapy plus EGFR-TKI for EGFR-mutated non-small cell lung cancer patients who develop brain metastasis. Arch Med Sci 2018;14:1298-307.

54. Costa DB, Shaw AT, Ou SH, Solomon BJ, Riely GJ, et al. Clinical experience with crizotinib in patients with advanced alk-rearranged non-small-cell lung cancer and brain metastases. J Clin Oncol 2015;33:1881-8.

55. Peters S, Camidge DR, Shaw AT, Gadgeel S, Ahn JS, et al. Alectinib versus crizotinib in untreated alk-positive non-small-cell lung cancer. N Engl J Med 2017;377:829-38.

56. Hida T, Nokihara H, Kondo M, Kim YH, Azuma K, et al. Alectinib versus crizotinib in patients with ALK-positive non-small-cell lung cancer (J-ALEX): an open-label, randomised phase 3 trial. Lancet 2017;390:29-39.

57. Gadgeel S, Peters S, Mok T, Shaw AT, Kim DW, et al. Alectinib versus crizotinib in treatment-naive anaplastic lymphoma kinase-positive $(\mathrm{ALK}+)$ non-small-cell lung cancer: CNS efficacy results from the ALEX study. Ann Oncol 2018;29:2214-22.

58. Kim DW, Tiseo M, Ahn MJ, Reckamp KL, Hansen KH, et al. Brigatinib in patients with crizotinib-refractory anaplastic lymphoma kinase-positive non-small-cell lung cancer: a randomized, multicenter phase ii trial. J Clin Oncol 2017;35:2490-8.

59. Gainor JF, Sherman CA, Willoughby K, Logan J, Kennedy E, et al. Alectinib salvages CNS relapses in ALK-positive lung cancer patients previously treated with crizotinib and ceritinib. J Thorac Oncol 2015;10:232-6.

60. Solomon BJ, Besse B, Bauer TM, Felip E, Soo RA, et al. Lorlatinib in patients with ALK-positive non-small-cell lung cancer: results from a global phase 2 study. Lancet Oncol 2018;19:1654-67.

61. Griesinger F, Roeper J, Pottgen C, Willborn KC, Eberhardt WEE. Brain metastases in ALK-positive NSCLC - time to adjust current treatment algorithms. Oncotarget 2018;9:35181-94. 
62. Lim SM, Kim HR, Lee JS, Lee KH, Lee YG, et al. Open-label, multicenter, phase ii study of ceritinib in patients with non-small-cell lung cancer harboring ros1 rearrangement. J Clin Oncol 2017;35:2613-8.

63. Solomon B, Shaw A, Ou S, Besse B, Felip E, et al. OA 05.06 phase 2 study of lorlatinib in patients with advanced ALK+/ROS1+ nonsmall-cell lung cancer. J Thorac Oncol 2017;12:S1756.

64. Weickhardt AJ, Scheier B, Burke JM, Gan G, Lu X, et al. Local ablative therapy of oligoprogressive disease prolongs disease control by tyrosine kinase inhibitors in oncogene-addicted non-small-cell lung cancer. J Thorac Oncol 2012;7:1807-14.

65. Gan GN, Weickhardt AJ, Scheier B, Doebele RC, Gaspar LE, et al. Stereotactic radiation therapy can safely and durably control sites of extra-central nervous system oligoprogressive disease in anaplastic lymphoma kinase-positive lung cancer patients receiving crizotinib. Int J Radiat Oncol Biol Phys 2014;88:892-8.

66. Johung KL, Yeh N, Desai NB, Williams TM, Lautenschlaeger T, et al. Extended survival and prognostic factors for patients with ALKrearranged non-small-cell lung cancer and brain metastasis. J Clin Oncol 2016;34:123-9. 